\title{
An analytical model for plug flow in microcapillaries with circular cross section
}

\author{
Zhizhao Che, Teck Neng Wong *, Nam-Trung Nguyen \\ School of Mechanical and Aerospace Engineering, Nanyang Technological University, 50 \\ Nanyang Avenue, Singapore 639798, Singapore \\ *Corresponding author. Tel.: +65 67905587; fax: +65 67911859. E-mail address: \\ mtnwong@ntu.edu.sg (T.N.Wong).
}

\begin{abstract}
Plug flow in microcapillaries or microchannels offers significant advantages for the development of microfluidic applications and recently triggers many interests and studies. Recirculation is formed within liquid plugs due to the presence of interfaces. This paper presents an analytical model to investigate the recirculation flow and the flow resistance in microcapillaries with circular cross section. A fourth order partial differential equation is used to model the Stokes flow within the liquid plug. The results of the flow field show that the flow pattern is affected by the plug length. The flow resistance is determined through the force balance of the liquid plug. The comparison of the flow field and the flow resistance from the analytical model and the experiments shows good agreement.
\end{abstract}

Keywords: Plug; Vortex; Flow resistance; Multiphase flow; Droplet-based microfluidics; Mathematical modelling

\section{Introduction}

The hydrodynamic behavior of plug flow in microscale is an important topic due to its wide applications in medicine (Fujioka et al., 2008), chemical reaction (Kreutzer et al., 2005b), detection and analysis. As the dimension of the systems shrinks, viscous force and surface tension force dominate the flow field, since the Reynolds number $\left(\mathrm{R}_{\mathrm{e}}\right)$ and the capillary number $\left(\mathrm{C}_{\mathrm{a}}\right)$ are both small in microscale (Squires and Quake, 2005). In a plug flow, which is also named bubble train, slug, segmented, Taylor flow, the bubbles have a capsular shape which completely or partially fill the channel cross-section (Angeli and Gavriilidis, 2008; Thulasidas et al., 1997). The presence of the interfaces induces recirculation in the liquid plug (Taylor, 1961). The circulated flow pattern offers many advantages over a single-phase flow. The heat and mass transfer processes (Handique and Burns, 2001; Nguyen and Wu, 2005; Song et al., 2003a) and chemical reaction (Song et al., 2006) can be significantly enhanced by folding and stretching the fluid. The axial dispersion and residual distribution time are greatly reduced (Teh et al., 2008; Trachsel et al., 2005) by confining reagents within the space of the liquid plug.

Determining the flow field in plug flow is an important but nontrivial task and recently triggered many interests and studies. Non-intrusive velocity measurement techniques were 
adopted to measure the velocity at a small scale, such as micro particle image velocimetry ( $\mu$ PIV) (Lindken et al., 2009; Sinton, 2004; Wereley and Meinhart, 2010). Although $\mu$ PIV has been widely used in the past decade, it is still a complex task to assess the multiphase flow by $\mu$ PIV. Refractive index of the different phases should be matched to ensure optical access without distortions caused by the fluid interface (Kim et al., 2004; Oishi et al., 2009). Particles should be carefully selected to guarantee a homogeneous and optimum solvability in either of the phases and to prevent agglomeration at the interface (Miessner et al., 2008).

The computational methods to simulate the flow field of a plug flow can be categorized into two types according to the interface shape. The first type, for simplicity and saving computation time, assumes the interface shape and reasonable results were obtained (Harries et al., 2003; Kashid et al., 2007; Tanthapanichakoon et al., 2006). The second type of computational method needs to use the interface predicting techniques such as front tracking method (Muradoglu et al., 2007), volume of fluid method (Scardovelli and Zaleski, 1999; Taha and Cui, 2004), level set method (Chen et al., 2009; Osher and Fedkiw, 2001), Lattice Boltzmann method (Yu et al., 2007; Zhang, 2010), and moving-grid method (Fujioka and Grotberg, 2004). These approaches are usually complex and computation-time consuming. This is unfavorable especially for applications in which additional processes need to analyze, such as chaos analysis in mixing process (Aref, 2002; Ottino, 1989; Song et al., 2003b). In contrast, with an explicit fully analytical model, analysis is much more convenient as it can be performed directly on the known flow fields.

Flow resistance is another significant aspect of plug flow. The pressure drop of plug flow is plug-length dependant (Horvath et al., 1973). Therefore, it can be used to estimate the plug length in opaque systems where it is difficult to determine the plug length through optical methods (Kreutzer et al., 2005a). A simple method to estimate the pressure drop is to use the Hagen-Poiseuille velocity profile and consider the friction on the wall of the channel. This method always underestimates the frictional resistance, as the recirculation effect at the end of the plug is neglected. However, the recirculation effect on the frictional resistance could be significant especially when the liquid plug is relatively short.

\begin{tabular}{|c|c|c|c|}
\hline \multicolumn{4}{|c|}{ Nomenclature } \\
\hline C & constant coefficient $(-)$ & $\varphi$ & stream function $\left(\mathrm{m}^{3} / \mathrm{s}\right)$ \\
\hline $\mathrm{Ca}$ & capillary number $(-)$ & & \\
\hline$C_{f}$ & plug resistance coefficient $(-)$ & Subscript & \\
\hline$f$ & Moody friction factor $(-)$ & adv & advancing end of the plug \\
\hline$F$ & force $(\mathrm{N})$ & center & vortex center \\
\hline$L$ & length of the plug (m) & $r$ & radial component \\
\hline$p$ & pressure $(\mathrm{Pa})$ & rec & receding end of the plug \\
\hline$r$ & radial coordinate in cylindrical coordinate system (m) & $\max$ & maximum of stream function \\
\hline$R$ & radius of the microcapillary $(\mathrm{m})$ & $\min$ & minimum of stream function \\
\hline $\operatorname{Re}$ & Reynolds number $(-)$ & $z$ & axial component \\
\hline$u$ & velocity $(\mathrm{m} / \mathrm{s})$ & & \\
\hline V & speed of the plug $(\mathrm{m} / \mathrm{s})$ & Overscrip & \\
\hline$z$ & axial coordinate in cylindrical coordinate system (m) & $\wedge$ & dimensionless variables \\
\hline Greek & & Abbreviat & tions \\
\hline$\theta$ & contact angle (rad) & $1 \mathrm{D}$ & one-dimelnsional \\
\hline$a$ & dynamic viscosity of fluid (Pa s) & 2D & two-dimensional \\
\hline$\sigma$ & surface tension $(\mathrm{N} / \mathrm{m})$ & $\mu \mathrm{PIV}$ & micro particle image velocimetry \\
\hline$\tau$ & stress $(\mathrm{Pa})$ & & \\
\hline
\end{tabular}


Microchannels or microcapillaries with circular cross section are widely used in both research studies and applications. However, the large curvature of the wall poses extra difficulty in optical measurement such as $\mu$ PIV. Special care should be taken when using this optical measurement method as light is refracted at the interface of materials with different refractive indices (Ahn et al., 2006). Although some experimental and numerical results of the pressure drop were reported (Adzima and Velankar, 2006; Chen et al., 2009; Kreutzer et al., 2005c), to our best knowledge, there is no analytical model reported to predict the flow fields and the flow resistance for plug flow in microcapillaries with circular cross section.

In this paper we present a two-dimensional (2D) analytical model for plug flow in microcapillaries or microchannels with circular cross section. The flow is rotational symmetric in a translating cylindrical coordinate system. In the next section, the flow field is modeled with a fourth order partial differential equation with the corresponding boundary conditions. Series solution is obtained, and is used to find the flow resistance of the plug. In Section 3, the flow field and the flow resistance are calculated and compared with experimental results from the literature (Ahn et al., 2006; Kreutzer et al., 2005c). The effects of plug length on the flow pattern and on the flow resistance are studied.

\section{Analytical model}

\subsection{Problem description and assumptions}

Fig. 1 shows liquid plugs in a microcapillary with circular cross section. The liquid plugs of deionized (DI) water are separated by air in the microcapillary. The radius of the capillary is $\mathrm{R}$. The plug, with its length $\mathrm{L}$, is moving to the right with a speed of $\mathrm{V}$.

In our analysis, the following assumptions are made.

(1)Stokes flow exists within the plug due to the low Reynolds number. The transient term and the convection term in the momentum equation are neglected.

(2)No body force is considered. The gravity is neglected and there is no other body force for the liquid plug.

(3)The interface is flat and is perpendicular to the wall of the capillary. Numerical simulations were performed to validate the flat interface assumption and to examine the effects of the contact angle and the curvature on the flow pattern in liquid plugs, and they are provided in Appendix A. (4)The liquid plug does not slip on the wall of the capillary. This is a reasonable assumption for liquid flow in microcapillaries/microchannels at micrometer scales. Some recent investigations showed that the wettability of the solid wall affects the slip length (Squires and Quake, 2005). Wetting (hydrophilic) surfaces obey the no-slip boundary condition as expected, whereas clean nonwetting (hydrophobic) surfaces exhibit apparent slip (Lumma et al., 2003; Priezjev et al., 2005). However, the slip length is still in the nanometer scale. For water flows over hydrophobic walls, the typical slip lengths that are observed range between 10 and $30 \mathrm{~nm}$ (Tabeling, 2009). For liquid flow in microchannels/ microcapillaries (normally tens to hundreds of micrometers), the liquid can be treated as a continuum and the no-slip condition applies.

(5) The viscosity of the adjacent immiscible phase is neglected and there is no shear stress on the free surface of the plug. This is a reliable assumption if the immiscible phase has a much lower viscosity than the liquid plug, such as gas.

(6) The liquid is an incompressible Newtonian fluid. 


\subsection{Flow field}

\subsubsection{Governing equation and boundary conditions}

We build a translating cylindrical coordinate system on the plug, as shown in Fig. 2. The plug takes the region of $0 \leqslant \mathrm{z} \leqslant \mathrm{L}, 0 \leqslant \mathrm{r} \leqslant \mathrm{R}$. The coordinate system is translating together with the plug at a speed of $\mathrm{V}$. In this way, the plug unit is relatively stationary with respect to the coordinate system, while the wall of the microcapillary is translating at a speed of $\mathrm{V}$ to the left. The middle cross section $\mathbf{X}-\mathbf{X}^{\prime}$ is indicated in Fig. 2.

For the plug at Stokes flow in a microcapillary with circular cross section, the governing equation is a fourth order partial differential equation (Deen, 1998; Duda and Vrentas, 1971; Meleshko, 2003; Shankar, 2007)

$$
E^{4} \varphi=0
$$

where $\varphi$, the stream function, is defined as

The operator $\mathrm{E}^{4}$ is

$$
u_{r}=-\frac{1}{r} \frac{\partial \varphi}{\partial z}, \quad u_{z}=\frac{1}{r} \frac{\partial \varphi}{\partial r}
$$

$$
E^{4} \equiv\left(\frac{\partial^{2}}{\partial^{2} r}-\frac{1}{r} \frac{\partial}{\partial r}+\frac{\partial^{2}}{\partial z^{2}}\right)\left(\frac{\partial^{2}}{\partial^{2} r}-\frac{1}{r} \frac{\partial}{\partial r}+\frac{\partial^{2}}{\partial z^{2}}\right)
$$

According to the boundary condition on the wall of the microcapillary, as shown in Fig. 2, we have

$$
\frac{1}{R} \frac{\partial \varphi(R, z)}{\partial r}=-V
$$

The two ends of the plug are free surfaces, as the shear effect of the adjacent gas is negligible

$$
\frac{\partial^{2}}{\partial z^{2}} \varphi(r, 0)=0, \quad \frac{\partial^{2}}{\partial z^{2}} \varphi(r, L)=0
$$

According to the definition of the stream function, the value of the stream function $\mathrm{u}$ is the same along the streamline. As the control volume of the liquid plug is closed with no fluid flowing in or out of the plug, the stream function is constant along the boundary of the plug. Here we set it to be zero

$$
\begin{array}{ll}
\varphi(r, 0)=0, & \varphi(r, L)=0 \\
\varphi(0, z)=0, & \varphi(R, z)=0
\end{array}
$$

Since the flow is rotational symmetric, and the radial velocity component $\mathrm{u}_{\mathrm{r}}$ is zero along the axis

$$
u_{r}(0, z)=\lim _{r \rightarrow 0}\left(-\frac{1}{r} \frac{\partial \varphi}{\partial z}\right)=0
$$

The fourth order partial differential Eq. (1) and its boundary condition Eqs. (5)- (8) represent a well-posed system of equations describing the spatial variation of the stream function in the liquid plug. Once the expression of the stream function is determined, the velocity, vorticity and stress can be determined from the stream function.

\subsubsection{Nondimensionalization}

The governing equation Eq. (1) and its boundary condition Eqs. (5)-(8) can be nondimensionalized for the convenience of modeling and analysis. The radius of the microcapillary $\mathrm{R}$ is set as the characteristic length

$$
\hat{r} \equiv \frac{r}{R}, \quad \hat{z} \equiv \frac{z}{R}, \quad \widehat{L} \equiv \frac{L}{R}
$$


where $\widehat{L}$ is the dimensionless plug length. The velocity of the liquid plug $\mathrm{V}$ is set as the characteristic speed. Hence the dimensionless velocity components in the $r$ and $z$ directions are respectively

$$
\hat{u}_{r} \equiv \frac{u_{r}}{V}, \quad \hat{u}_{z} \equiv \frac{u_{z}}{V}
$$

According to the definition of the stream function in Eq. (2), the dimensionless stream function can be defined as

$$
\hat{\varphi} \equiv \frac{\varphi}{V R^{2}}
$$

Therefore, the dimensionless governing equation can be obtained from Eq. (3)

$$
\left(\frac{\partial^{2}}{\partial \hat{r}^{2}}-\frac{1}{\hat{r}} \frac{\partial}{\partial \hat{r}}+\frac{\partial^{2}}{\partial \hat{z}^{2}}\right)\left(\frac{\partial^{2}}{\partial \hat{r}^{2}}-\frac{1}{\hat{r}} \frac{\partial}{\partial \hat{r}}+\frac{\partial^{2}}{\partial \hat{z}^{2}}\right) \hat{\varphi}=0
$$

The corresponding dimensionless boundary conditions can be obtained from Eqs. (4)- (7)

$$
\begin{aligned}
& \frac{\partial \hat{\varphi}}{\partial \hat{r}}(1, \hat{z})=-1 \\
& \frac{\partial^{2}}{\partial \hat{z}^{2}} \varphi(\hat{r}, 0)=0, \quad \frac{\partial^{2}}{\partial \hat{z}^{2}} \hat{\varphi}(\hat{r}, \widehat{L})=0 \\
& \hat{\varphi}(0, \hat{z})=0, \quad \hat{\varphi}(1, \hat{z})=0 \\
& \hat{\varphi}(\hat{r}, 0)=0, \quad \hat{\varphi}(\hat{r}, \hat{L})=0
\end{aligned}
$$

\subsubsection{Analytical solution}

According to the fact that the governing equation [Eq. (12)] is linear and the domain is rectangular, a tentative solution is of a series form (Deen, 1998; Duda and Vrentas, 1971; Shankar, 2007). We can construct the format of the solution of the governing equation [Eq. (12)] as

$$
\hat{\varphi}(\hat{r}, \hat{z})=\sum_{n=1}^{\infty} \sin \left(n \pi \frac{\hat{z}}{\hat{L}}\right) A_{n} F_{n}
$$

where

$$
F_{n}(\hat{r})=\frac{\hat{r} I_{1}(n \pi \hat{r} / \hat{L}) I_{2}(n \pi / \widehat{L})-\hat{r}^{2} I_{1}(n \pi / \widehat{L}) I_{2}(n \pi \hat{r} / \widehat{L})}{I_{2}^{2}(n \pi / \widehat{L})}
$$

where $I_{n}$ is the modified Bessel function of the first kind of order $n$. This format of solution in Eq. (17) satisfies the dimensionless governing equation [Eq. (12)], as well as the boundary condition of $\hat{\varphi}=0$ in Eqs. (15) and (16). It also satisfies the free surface boundary condition Eq. (14). Hence, the coefficient $A_{n}$ can be obtained which lets Eq. (17) satisfy the boundary velocity on the wall Eq. (13). From Eq. (13), we have

where

$$
-1=\sum_{n=1}^{\infty} \sin \left(n \pi \frac{\hat{z}}{\hat{L}}\right) A_{n} F_{n}^{\prime}(1)
$$

$$
F_{n}^{\prime}(\hat{r})=\frac{-n \pi \hat{r}^{2} I_{1}(n \pi / \widehat{L}) I_{1}(n \pi \hat{r} / \widehat{L})+n \pi \hat{r} I_{0}(n \pi \hat{r} / \widehat{L}) I_{2}(n \pi / \widehat{L})}{\widehat{L} I_{2}^{2}(n \pi / \widehat{L})}
$$

Multiplying $\sin (n \pi \hat{z} / \widehat{L})$ on both sides of Eq. (19) and integrating with respect to z from 0 to $\widehat{L}$, we can obtain the coefficient $A_{n}$ through the Fourier transform

$$
A_{n}=\frac{2\left[(-1)^{n}-1\right]}{F_{n}^{\prime}(1) n \pi}
$$


Through this way, the stream function field is established. The velocity field can also be obtained from the stream function

$$
\begin{aligned}
& \hat{u}_{r}=-\frac{1}{\hat{r}} \frac{\partial}{\partial z} \hat{\varphi}(\hat{r}, \hat{z})=-\sum_{n=1}^{\infty} \frac{n \pi}{\widehat{L}} \cos \left(n \pi \frac{z}{\hat{L}}\right) A_{n} \frac{F_{n}}{\hat{r}} \\
& u_{z}=\frac{1}{\hat{r}} \frac{\partial}{\partial \hat{r}} \hat{\varphi}(\hat{r}, \hat{z})=\sum_{n=1}^{\infty} \sin \left(n \pi \frac{\hat{z}}{\hat{L}}\right) A_{n} \frac{F_{n}^{\prime}(\hat{r})}{\hat{r}}
\end{aligned}
$$

\subsection{Flow resistance of the liquid plug}

The force balance diagram for the liquid plug is illustrated in Fig. 3. These forces are in balance, as inertial effect can be neglected due to the low Reynolds number. The frictional force can be derived by integrating the shear stress along the walls of the microcapillary.

$$
\begin{aligned}
F_{f} & =-2 \pi R \int_{0}^{L} \tau_{r z} d z=-2 \pi R \mu \int_{0}^{L}\left[\frac{\partial}{\partial r}\left(\frac{1}{r} \frac{\partial}{\partial r} \varphi\right)\right] d z \\
& =-2 \mu V R \widehat{L} \sum_{n=1}^{\infty} \frac{1-(-1)^{n}}{n} A_{n} Q(1)
\end{aligned}
$$

Where

$$
Q(1)=-\frac{2 n \pi I_{1}(n \pi / \widehat{L})^{2}}{\widehat{L} I_{2}(n \pi / \widehat{L})^{2}}
$$

Although we assume flat interfaces (assumption 3 in Section 2.1) in obtaining the flow field, the effects of surface tension and contact angle hysteresis on the flow resistance can be included in the force balance. The surface tension and the contact angle influence the plug speed, while the plug speed affects the flow field in the plug. In this way, the effects of the surface tension and contact angle on the flow field can be considered indirectly through the plug speed. The forces due to surface tension at the advancing and receding ends are respectively

$$
\begin{aligned}
& F_{\mathrm{adv}}=2 \pi R \sigma_{\mathrm{adv}} \cos \left(\theta_{\mathrm{adv}}\right) \\
& F_{\text {rec }}=2 \pi R \sigma_{\text {rec }} \cos \left(\theta_{\text {rec }}\right)
\end{aligned}
$$

where $\theta_{\text {adv }}$ and $\theta_{\text {rec }}$ are the advancing and receding contact angles, respectively. $\sigma_{\text {adv }}$ and $\sigma_{\text {rec }}$ denote the surface tension at the advancing and receding ends, respectively. For different materials of fluids and of the capillaries, the contact angles and surface tensions can be different case by case. They can be obtained experimental measurements or from handbooks (De Gennes, 1985; Lide and Haynes, 2010).

The force balance equation of the liquid plug is

$$
\Delta p \pi R^{2}=F_{f}+F_{\text {rec }}-F_{\text {adv }}
$$

where $\Delta \mathrm{p}=\mathrm{p}_{\mathrm{rec}}-\mathrm{p}_{\mathrm{adv}}$ is the pressure drop from the receding end to the advancing end of the plug. This leads to the driving pressure

$$
\begin{aligned}
\Delta p= & -\frac{2 \mu V \widehat{L}}{\pi R} \sum_{n=1}^{\infty} \frac{1-(-1)^{n}}{n} A_{n} Q(1) \\
& +\frac{2}{R}\left[\sigma_{\text {rec }} \cos \left(\theta_{\text {rec }}\right)-\sigma_{\text {adv }} \cos \left(\theta_{\text {adv }}\right)\right]
\end{aligned}
$$

In (29), the first term is due to shear resistance, and the second term is due to surface tension.

The pressure difference in Eq. (29) can be nondimensionalized to plug resistance coefficient $\mathrm{C}_{\mathrm{f}}$, which is defined as the product of the Moody friction factor $\mathrm{f}$ and the Reynolds number $\mathrm{R}_{\mathrm{e}}$. 
The characteristic velocity is the speed of the plug $\mathrm{V}$, and the characteristic length is the radius of the mirocapillary $\mathrm{R}$

$$
\begin{aligned}
& f=\frac{2 R \Delta p}{L \rho V^{2}} \\
& R e=\frac{\rho V R}{\mu}
\end{aligned}
$$

Hence, the plug resistance coefficient $\mathrm{C}_{\mathrm{f}}$ can be obtained from Eq. (29)

$$
\begin{aligned}
C_{f}= & f R e \\
= & -\frac{4}{\pi} \sum_{n=1}^{\infty} \frac{1-(-1)^{n}}{n} A_{n} Q(1) \\
& +\frac{4\left[\sigma_{\mathrm{rec}} \cos \left(\theta_{\mathrm{rec}}\right)-\sigma_{\mathrm{adv}} \cos \left(\theta_{\mathrm{adv}}\right)\right]}{\widehat{L} \mu \mathrm{V}}
\end{aligned}
$$

\section{Results and discussion}

\subsection{Flow field}

\subsubsection{Comparison between $2 D$ model with experimental results}

When a liquid plug moves through in a microcapillary, in the presence of the interfaces at the advancing and receding ends of the liquid plugs, the flow pattern is much different from that of the continuous flow. For a typical dimensionless plug length $\widehat{L}=2.45$, the vortices of toroidal shape are shown in Fig. 4a, and the streamlines and the velocity vectors on a longitudinal section are shown in Fig. $4 \mathrm{~b}$ and c respectively. When the liquid in the plug is moving towards the advancing end, it is blocked by the advancing interface and moves towards the wall of the microcapillary. Due to the presence of the receding interface, liquid moves from the wall of the microcapillary towards the center of the plug. This movement of the fluid produces recirculation in the liquid plug with respect to the translating frame of reference, which is clearly shown in Fig. 4a-c. A streamline $\mathrm{S}-\mathrm{S}$ along the $\mathrm{z}$-axis works as the axis of the toroidal-shaped vortex. The comparison of velocity component between the analytical result and the measured flow field by Ahn et al. (2006) is shown in Fig. 4d and e respectively. The measured flow field is obtained with frequency domain optical coherence tomography. Fig. $4 \mathrm{~d}$ and e show the velocity component projected in the direction which is tilted $1.5^{\circ}$ away from the cross-sectional plane of the microcapillary. The dimensionless plug length $\widehat{L}=2.45$ is obtained in pixel from the image by a Matlab program, where

$$
\widehat{L}=\frac{\text { area of plug in pixel }}{{\text { (width of plug in pixel })^{2}}^{2}}
$$

The comparison with the experimental result shows that the analytical model can offer a reasonable good prediction of the flow field within liquid plugs.

\subsubsection{Vortex centers}

As the stream function at the boundary is zero, the maximum/ minimum points of the stream function( $\hat{\varphi}_{\max }$ or $\hat{\varphi}_{\min }$ ) within the plug correspond to the vortex centers, and the magnitude of the stream function reflects the strength of the vortex. Hence the location of the vortex center and the value of the stream function at the vortex center are important parameters to quantify the vortex pattern in the plug. As the stream function is symmetric with respect to the cross section 
$\mathbf{X}-\mathbf{X}^{\prime}$ (shown in Fig. 2), the vortex centers are located along $\mathbf{X}-\mathbf{X}^{\prime}$. The radial position of the vortex center ( $\hat{r}_{\text {center }}$ ) for different dimensionless plug lengths $\widehat{L}$ and the corresponding dimensionless stream function ( $\hat{\varphi}_{\text {center. }}$ ) are shown in Fig. 5 . The location of the vortex centers and the strength of vortices strongly depend on the dimensionless plug length $\widehat{L}$. The vortex center moves in the direction towards the axis of the microcapillary, while $\hat{\varphi}_{\text {center increases when }}$ the dimensionless plug length $\widehat{L}$ is increased. This effect can be observed in Fig. 6. Initially, with the increase of plug length, the vortices increase in size and strength, and the vortex centers move rapidly in the direction towards streamline S-S. The magnitude of $\hat{\varphi}_{\text {center increases as the }}$ vortices expand. For relatively large dimensionless plug length $\left(\hat{L}>\widehat{L}_{\text {long }}\right)$ the vortex centers

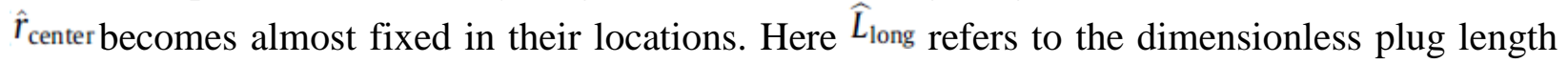
where the velocity profile $\hat{u}_{z}$ along $\mathbf{X}-\mathbf{X}$ ' approaches the fully developed velocity profile as shown in Fig. 7, which will be explained in Section 3.1.3. The unchanged velocity profiles make the locations of the vortex centers nearly constant.

If the length of the liquid plug approaches infinity $(\widehat{L} \rightarrow \infty)$, the flow can be simplified to the one-dimensional (1D) flow. For a single phase flow, the fully developed velocity profile in a straight tube is parabolic, and the axial pressure gradient $\Delta p / L$ is independent of $\mathrm{r}$

$$
u_{z, 1}^{\prime}=\frac{\Delta p}{4 L \mu}\left(R^{2}-r^{2}\right)
$$

The mean velocity can be obtained through integration

$$
V=\frac{\Delta P R^{2}}{8 L \mu}
$$

As a result, the dimensionless velocity profile in the translating coordinate system is

$$
\hat{u}_{z, 1}=\frac{u_{z, 1}^{\prime}-V}{V}=1-2 \hat{r}^{2}
$$

According to the dimensionless group, the dimensionless stream function follows

$$
\hat{u}_{z}=\frac{1}{\hat{r}} \frac{\partial \hat{\varphi}}{\partial \hat{r}}
$$

Being similar to the stream function boundary condition of plug in Eq. (15), the boundary condition for the stream function of the 1D single phase flow in a microcapillary is

$$
\hat{\varphi}(0)=0, \quad \hat{\varphi}(1)=0
$$

With the velocity profile in Eq. (36), the dimension stream function can be obtained by integration with the boundary condition in Eq. (38). And it reads

$$
\hat{\varphi}_{1 D}(\hat{r})=\frac{1}{2}\left(\hat{r}^{2}-\hat{r}^{4}\right)
$$

The vortex center appears at the minimum/maximum point of the dimensionless stream function. In our case of $\hat{\varphi}_{1 D}(\hat{r})$, the maximum value of the dimensionless stream function is

$\hat{\varphi}_{\text {center }}=0.125$ which appears at $\hat{r}_{\text {center }}=\sqrt{2} / 2$. This is exactly the asymptotic limit of the stream function an the asymptotic location of the vortex center as shown in Fig. 5. This can be regarded as a validation of our 2D model.

\subsubsection{Velocity profiles and validation by $1 D$ model}

The middle cross section $\mathbf{X}-\mathbf{X}$ ' is selected as a typical cross section for analysis of the velocity profile in the liquid plug. Fig. 7a compares the 1D solution in Eq. (36) with the analytical result in Eq. (23) at cross section $\mathbf{X}-\mathbf{X}^{\prime}$ from the proposed 2D analytical model. When the length of the 
liquid plug approaches infinity, the $2 \mathrm{D}$ solution reduces to the $1 \mathrm{D}$ solution (such as $\widehat{L}=100$ in Fig. 7a). For a plug with $\widehat{L}=2$ (the length of the plug $\mathrm{L}$ is twice of the radius), the predicted velocity profile from the $2 \mathrm{D}$ model shows a $4 \%$ discrepancy with the $1 \mathrm{D}$ solution at point $\hat{r}=0$. The $4 \%$ discrepancy does not mean that the flow in the plug is similar to the $1 \mathrm{D}$ flow even $\widehat{L}>2$. In plug flow, as predicted by the 2D model, due to the presence of the advancing interface, liquid moves from the center of the plug towards the wall, while due to the presence of the receding interface, liquid moves from the wall to the center of the plug. Therefore, the effect of the advancing/ receding interface on the flow field near the ends of the plug is expected to be more significant than that in the middle cross section of the plug (section $\mathrm{X}-\mathrm{X}^{\prime}$ ).

Fig. $7 \mathrm{~b}$ shows the axial component of velocity profiles $\hat{u}_{z}$ for different plug lengths $\widehat{L}$ along cross section $X-X$ '. For long plugs $\widehat{L}=2$ in Fig. 7 b), the velocity profile is approximately fully developed. For short plugs $(\widehat{L}=0.5$ in Fig. $7 \mathrm{~b})$, there are two local maximum points of the velocity field, as vortices move towards the wall of the microcapillary.

\subsection{The plug resistance coefficient}

According to Eq. (32), the plug resistance coefficient $C_{f}$ is influenced by the shear force and the surface tension. The contribution of the shear force (first term on the right hand side of Eq. (32)) depends on the infinite series solution. The surface tension contribution (second term on the right hand side of Eq. (32)) depends on the fluid viscosity $\mu$, plug speed $V_{\text {avg }}$, contact angles $\theta_{\text {adv }}$ and $\theta_{\text {rec }}$, and surface tension $\sigma$.

Fig. 8 shows the variation of the plug resistance coefficient $C_{f}$ with the increase of the dimensionless plug length $\widehat{L}$. The experimental results for different air/liquid plug systems by Kreutzer et al. (2005c) are also shown. However, to compare the analytical results under the experimental condition, some parameters $\left(\theta_{\mathrm{adv}}, \theta_{\text {rec }}\right.$ and $\left.\mathrm{V}\right)$ remain unknown for the surface tension contribution in Eq. (32). To consider the contribution of the surface tension force in the model, here, we introduce a coefficient $\mathrm{C}$ in Eq. (32)

$$
C=\frac{4\left[\sigma_{\text {rec }} \cos \left(\theta_{\text {rec }}\right)-\sigma_{\text {adv }} \cos \left(\theta_{\text {adv }}\right)\right]}{\mu V}
$$

Therefore, we can simplify Eq. (32) into

$$
C_{f}=-\frac{4}{\pi} \sum_{n=1}^{\infty} \frac{1-(-1)^{n}}{n} A_{n} Q(1)+\frac{C}{\widehat{L}}=\left(C_{f}\right)_{\text {friction }}+\left(C_{f}\right)_{\sigma}
$$

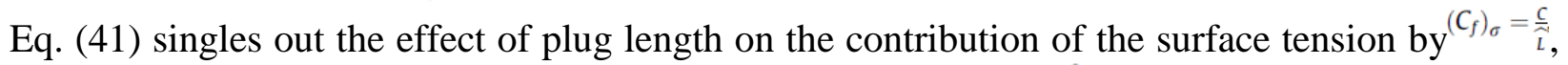
which is inversely proportional to the dimensionless plug length $\widehat{L}$. This is because the surface tension occurs at the ends of the plug, while the plug resistance coefficient considers the resistance per unit length through average.

The value of the coefficient $\mathrm{C}$ in Eq. (40) for three experimental fluids can be fitted by the method of least squares from the experimental results in Eq. (41), and then the coefficient $\mathrm{C}$ is substituted into Eq. (41) to calculate the total plug resistance coefficient $\mathrm{C}_{\mathrm{f}}$. The results from the model for three experimental fluids are shown in Fig. 8. From the comparison, we can see that our analytical model agrees well with the experimental data for different air/liquid plug systems. According to Eq. (40), when there is no external effect to change the surface tension such as by thermocapillarity (Burns et al., 1996), electrowetting (Mugele and Baret, 2005) and gradients of surfactant concentration, the surface tensions at the advancing end and at the receding end are 
identical, i.e., $\sigma_{\mathrm{adv}}=\sigma_{\mathrm{rec}}$. Therefore, the constant coefficient $\mathrm{C}$ in Eq. (40) is proportional to surface tension and inversely proportional to the viscosity, $C \propto(\sigma / \mu)$. The surface tension and the viscosity of the three experimental fluids are $\sigma_{\text {water }}=73 \mathrm{mN} / \mathrm{m}, \sigma_{\text {decane }}=24 \mathrm{mN} / \mathrm{m}, \sigma_{\text {tetradecane }}=$ $26 \mathrm{mN} / \mathrm{m}$, and $\mu_{\text {water }}=1.01 \mathrm{mPa} \mathrm{s}, \mu_{\text {decane }}=0.924 \mathrm{mPa} \mathrm{s}, \mu_{\text {tetradecane }}=2.32 \mathrm{mPa} \mathrm{s}$, respectively. Therefore, the ratios $\sigma / \mu$ for three fluids are

$$
(\sigma / \mu)_{\text {water }}:(\sigma / \mu)_{\text {decane }}:(\sigma / \mu)_{\text {tetradecane }}=72: 26: 11=6.4
$$

$$
: 2.3: 1
$$

Meanwhile, the coefficients fitted by the method of least squares for the three experimental fluids are

$$
C_{\text {water }}: C_{\text {decane }}: C_{\text {tetradecane }}=135: 83.8: 23.8=5.7: 3.5: 1
$$

This agreement to $C \propto(\sigma / \mu)$ is satisfactory.

Fig. 8 shows the effect of the dimensionless plug length on the plug resistance coefficient. As the dimensionless plug length $\widehat{L}$ increases, plug resistance coefficient $C_{\mathrm{f}}$ decreases dramatically. This can be explained by the fact that the high velocity gradient at the end of the plug dominate the flow in short plugs, which causes large flow resistance. Hence short plugs experience a larger resistance coefficient. As the dimensionless plug length $\widehat{L}$ increases, plug resistance coefficient $\mathrm{C}_{\mathrm{f}}$ decreases gradually. For a long plug such as $\widehat{L}=20$, the region near the front/rear interface is still significantly affected, while the effect on the region near the center is insignificant. The high velocity gradient at the end of the plug significantly increases the local shear stress on the wall of the microcapillary. Therefore, it requires an extremely long plug to "dilute" the effect of the end on the plug resistance coefficient.

As the dimensionless plug length further increases, the value of $\mathrm{C}_{\mathrm{f}}$ gradually approaches an asymptotic limit and it remains above this limit. The dashed horizontal line with $\mathrm{C}_{\mathrm{f}}=16$ is plotted in Fig. 8 to show that the plug resistance coefficient approaches 16 as the dimensionless plug length $\widehat{L}$ approaches infinity, which is exactly the resistance coefficient for single phase fully developed laminar flow in a capillary (White, 2002). Using the 2D model, the plug resistance coefficients are always larger than 16 , and this value is the asymptotic limit when $\widehat{L} \rightarrow \infty$, as shown in Fig. 8. For example, for $C_{f}=16.16$ (that is $1 \%$ deviation from $C_{f}=16$ ), the corresponding dimensionless plug length is $\widehat{L}=24$.6. Under this condition, the plug behaves as if it is the fully developed continuous flow. Therefore, for $\widehat{L}<24.6$, the idealization of a liquid plug as fully developed continuous flow cannot provide an accurate prediction of the pressure loss.

\section{Conclusions}

An analytical model is presented in this paper to investigate the plug flow in microcapillaries with circular cross section. The Stokes flow field in the liquid plug is modeled with a fourth order partial differential equation with its boundary conditions. Series solution of the governing equation is obtained and the boundary conditions are satisfied. The velocity field is utilized to find the flow resistance of the liquid plug.

The flow pattern within the liquid plug is characterized by the vortex center. The results from the analytical model show that the vortex center shift towards the wall of the microcapillary as the plug length decreases. The results also show the dependency of the plug resistance on the plug length. For short plugs, the plug resistance coefficient increase dramatically as the 
dimensionless length decreases. For long plugs and as the dimensionless plug length approaches infinity, the plug resistance coefficient approaches 16, which is the resistance coefficient of continuous laminar flow in a cylindrical tube. 


\section{Appendix A. Effects of contact angles and curved interface shape on the flow pattern}

To validate the flat interface assumption in Section 2.1, and to examine the effects of the contact angle and the curvature on the flow pattern in liquid plugs, we carried out numerical simulation using Fluent 6.3.26 with the finite volume method (FVM). Different contact angles were specified and interface shapes of spherical caps were assumed, as shown in Fig. 9. The result with a flat interface has a contact angle $\theta=90^{\circ}$, as shown in Fig. 9i. When the contact angle is slightly changed $\theta=90^{\circ} \pm 22.5^{\circ}$, the flow pattern within the liquid plug is almost identical, as shown in Fig. 9g and k. If the contact angle changes further $\theta=90^{\circ} \pm 45^{\circ}$, the curvature of the interfaces increases. The flow in the plug is still very similar to the case with flat interfaces, as shown in Fig. 9e and $\mathrm{m}$. If the contact angle changes to an extreme condition $\theta=0^{\circ}$, the wall is superhydrophilic, and the interface is highly curved, as shown in Fig. 9a. The other extreme condition of contact angle is $\theta=180^{\circ}$, where the wall is superhydrophobic. At this condition, the flow near the spherical cap of the interface is slow (because the corresponding streamlines are sparse), as shown in Fig. 9q. However, the flow is dominated by the counter rotating vortices. This is a significant feature of plug flow and is captured with flat-interface assumption. Therefore, the proposed model can be used for a group of plug flow in microchannels where the contact angle is not far from $90^{\circ}$. 


\section{References}

[1] Adzima, B.J., Velankar, S.S., 2006. Pressure drops for droplet flows in microfluidic channels. J. Micromech. Microeng. 16, 1504.

[2] Ahn, Y.-C., Jung, W., Chen, Z., 2006. Turbid two-phase slug flow in a microtube: Simultaneous visualization of structure and velocity field. Appl. Phys. Lett. 89, 064109.

[3] Angeli, P., Gavriilidis, A., 2008. Hydrodynamics of Taylor flow in small channels: a review. P. I. Mech. Eng. C - J. Mech. 222, 737-751.

[4] Aref, H., 2002. The development of chaotic advection. Phys. Fluids 14, 1315-1325.

[5] Burns, M.A., Mastrangelo, C.H., Sammarco, T.S., Man, F.P., Webster, J.R., Johnson, B.N., Foerster, B., Jones, D., Fields, Y., Kaiser, A.R., Burke, D.T., 1996. Microfabricated structures for integrated DNA analysis. Proc. Natl. Acad. Sci. US Am. 93, 5556-5561.

[6] Chen, Y., Kulenovic, R., Mertz, R., 2009. Numerical study on the formation of Taylor bubbles in capillary tubes. Int. J. Therm. Sci. 48, 234-242.

[7] De Gennes, P.G., 1985. Wetting: statics and dynamics. Rev. Mod. Phys. 57, 827-863.

[8] Deen, W.M., 1998. Analysis of Transport Phenomena. Oxford University Press, New York.

[9] Duda, J.L., Vrentas, J.S., 1971. Steady flow in the region of closed streamlines in a cylindrical cavity. J. Fluid Mech. 45, 247-260.

[10] Fujioka, H., Grotberg, J.B., 2004. Steady propagation of a liquid plug in a two-dimensional channel. J. Biomech. Eng. 126, 567-577.

[11] Fujioka, H., Takayama, S., Grotberg, J.B., 2008. Unsteady propagation of a liquid plug in a liquid-lined straight tube. Phys. Fluids 20, 062104.

[12] Handique, K., Burns, M.A., 2001. Mathematical modeling of drop mixing in a slit-type microchannel. J. Micromech. Microeng. 11, 548-554.

[13] Harries, N., Burns, J.R., Barrow, D.A., Ramshaw, C., 2003. A numerical model for segmented flow in a microreactor. Int. J. Heat Mass. Tran. 46, 3313-3322.

[14] Horvath, C., Solomon, B.A., Engasser, J.M., 1973. Measurement of radial transport in slug flow using enzyme tubes. Ind. Eng. Chem. Fund. 12, 431-439.

[15] Kashid, M.N., Agar, D.W., Turek, S., 2007. CFD modelling of mass transfer with and without chemical reaction in the liquid-liquid slug flow microreactor. Chem. Eng. Sci. 62, 51025109. 
[16] Kim, B.J., Liu, Y.Z., Sung, H.J., 2004. Micro PIV measurement of two-fluid flow with different refractive indices. Meas. Sci. Technol. 15, 1097-1103.

[17] Kreutzer, M.T., Eijnden, M.G.V.D., Kapteijn, F., Moulijn, J.A., Heiszwolf, J.J., 2005a. The pressure drop experiment to determine slug lengths in multiphase monoliths. Catal. Today 105, $667-672$.

[18] Kreutzer, M.T., Kapteijn, F., Moulijn, J.A., Heiszwolf, J.J., 2005b. Multiphase monolith reactors: chemical reaction engineering of segmented flow in microchannels. Chem. Eng. Sci. 60, 5895-5916.

[19] Kreutzer, M.T., Kapteijn, F., Moulijn, J.A., Kleijn, C.R., Heiszwolf, J.J., 2005c. Inertial and interfacial effects on pressure drop of Taylor flow in capillaries. AIChE J. 51, 2428-2440.

[20] Lide, D.R., Haynes, W.M., 2010. CRC Handbook of Chemistry and Physics, Internet Version 2010, nineteenth ed. CRC Press, Cleveland, Ohio.

[21] Lindken, R., Rossi, M., Große, S., Westerweel, J., 2009. Micro-particle image velocimetry (PIV): recent developments, applications, and guidelines. Lab. Chip 9, 2551-2567.

[22] Lumma, D., Best, A., Gansen, A., Feuillebois, F., Rädler, J.O., Vinogradova, O.I., 2003. Flow profile near a wall measured by double-focus fluorescence cross-correlation. Phys. Rev. E $67,056313$.

[23] Meleshko, V.V., 2003. Selected topics in the history of the two-dimensional biharmonic problem. Appl. Mech. Rev. 56, 33-85.

[24] Miessner, U., Lindken, R., Westerweel, J., 2008. 3D - velocity measurements in microscopic two-phase flows by means of micro PIV. In: 14th Int. Symp. on Applications of Laser Techniques to Fluid Mechanics, Lisbon, Portugal, pp. 1-8.

[25] Mugele, F., Baret, J.C., 2005. Electrowetting: from basics to applications. J. Phys. Condens. Mater. 17, R705-R774.

[26] Muradoglu, M., Günther, A., Stone, H.A., 2007. A computational study of axial dispersion in segmented gas-liquid flow. Phys. Fluids 19, 072109.

[27] Nguyen, N.-T., Wu, Z., 2005. Micromixers-a review. J. Micromech. Microeng. 15, R1R16.

[28] Oishi, M., Kinoshita, H., Fujii, T., Oshima, M., 2009. Confocal micro-PIV measurement of droplet formation in a T-shaped micro-junction. J. Phys. Conf. Ser., 012061.

[29] Osher, S., Fedkiw, R.P., 2001. Level set methods: an overview and some recent results. J. Comput. Phys. 169, 463-502.

[30] Ottino, J.M., 1989. The Kinematics of Mixing: Stretching, Chaos, and Transport. Cambridge University Press, New York. 
[31] Priezjev, N.V., Darhuber, A.A., Troian, S.M., 2005. Slip behavior in liquid films on surfaces of patterned wettability: comparison between continuum and molecular dynamics simulations. Phys. Rev. E 71, 041608.

[32] Scardovelli, R., Zaleski, S., 1999. Direct numerical simulation of free-surface and interfacial flow. Annu. Rev. Fluid Mech. 31, 567-603.

[33] Shankar, P.N., 2007. Slow Viscous Flows: Qualitative Features and Quantitative Analysis Using Complex Eigenfunction Expansions. Imperial College Press.

[34] Sinton, D., 2004. Microscale flow visualization. Microfluid. Nanofluid. 1, 2-21.

[35] Song, H., Bringer, M.R., Tice, J.D., Gerdts, C.J., Ismagilov, R.F., 2003a. Experimental test of scaling of mixing by chaotic advection in droplets moving through microfluidic channels. Appl. Phys. Lett. 83, 4664-4666.

[36] Song, H., Chen, D.L., Ismagilov, R.F., 2006. Reactions in droplets in microfluidic channels. Angew. Chem. Int. Edit. 45, 7336-7356.

[37] Song, H., Tice, J.D., Ismagilov, R.F., 2003b. A microfluidic system for controlling reaction networks in time. Angew. Chem. Int. Edit. 42, 768-772.

[38] Squires, T.M., Quake, S.R., 2005. Microfluidics: fluid physics at the nanoliter scale. Rev. Mod. Phys. 77, 977-1026.

[39] Tabeling, P., 2009. A brief introduction to slippage, droplets and mixing in microfluidic systems. Lab. Chip 9, 2428-2436.

[40] Taha, T., Cui, Z.F., 2004. Hydrodynamics of slug flow inside capillaries. Chem. Eng. Sci. 59, 1181-1190.

[41] Tanthapanichakoon, W., Aoki, N., Matsuyama, K., Mae, K., 2006. Design of mixing in microfluidic liquid slugs based on a new dimensionless number for precise reaction and mixing operations. Chem. Eng. Sci. 61, 4220-4232.

[42] Taylor, G.I., 1961. Deposition of a viscous fluid on the wall of a tube. J. Fluid. Mech. 10, 161-165.

[43] Teh, S.Y., Lin, R., Hung, L.H., Lee, A.P., 2008. Droplet microfluidics. Lab. Chip 8, 198220.

[44] Thulasidas, T.C., Abraham, M.A., Cerro, R.L., 1997. Flow patterns in liquid slugs during bubble-train flow inside capillaries. Chem. Eng. Sci. 52, 2947-2962.

[45] Trachsel, F., Günther, A., Khan, S., Jensen, K.F., 2005. Measurement of residence time distribution in microfluidic systems. Chem. Eng. Sci. 60, 5729-5737. 
[46] Wereley, S.T., Meinhart, C.D., 2010. Recent advances in micro-particle image velocimetry. Annu. Rev. Fluid Mech. 42, 557-576.

[47] White, F.M., 2002. Fluid Mechanics, 5th ed. McGraw-Hill, Boston.

[48] Yu, Z., Hemminger, O., Fan, L.-S., 2007. Experiment and lattice Boltzmann simulation of two-phase gas-liquid flows in microchannels. Chem. Eng. Sci. 62, 7172-7183.

[49] Zhang, J., 2010. Lattice Boltzmann method for microfluidics: models and applications. Microfluid. Nanofluid. 10, 1-28. 


\section{List of Figures}

Fig. 1 Typical liquid plugs in a microcapillary with circular cross section. The liquid plugs of deionized (DI) water are separated by air in a glass microcapillary of inner radius $176 \mu \mathrm{m}$ (Sigma-Aldrich, P0549) viewed under an inverted microscope.

Fig. 2 Mathematical modelling of a plug moving in a microcapillary. With the translating frame of reference fixed on the plug unit, the wall is moving towards left at a speed of $\mathrm{V}$.

Fig. 3 Force balance diagram of the liquid plug moving towards right in a microcapillary at a speed of $\mathrm{V}$.

Fig. 4 Flow pattern within a liquid plug of dimensionless plug length $\widehat{L}=2.45$. (a) Three-dimensional view of the vortices of toroidal shape within a liquid plug in a capillary of circular cross section. The fluid flows along the iso-surfaces of stream function, and forms vortices of toroidal shape. (b) Stream function contours, the increment between two consecutive contours is $\Delta \hat{\varphi}=0.02$. The crosses and the corresponding values indicate the locations and stream functions of the vortex centers, respectively. (c) Velocity vectors. (d) Dimensionless velocity projected in the direction $\theta=1.5^{\circ}$ from the $2 \mathrm{D}$ model. (e) Experimental result of velocity projected on the direction $\theta=1.5^{\circ}$ (Ahn et al., 2006), the unit is $\mathrm{mm} / \mathrm{s}$ (reproduced with permission).

Fig. 5 Radial position of the vortex center for different dimensionless plug lengths and the corresponding stream functions.

Fig. 6 Stream function contours within liquid plugs of different plug lengths. (a) $\widehat{L}=1$, (b) $\widehat{L}=1.5$ and (c) $\widehat{L}=2$. The increment between two consecutive contours is $\Delta \hat{\varphi}=0.02$. The crosses and corresponding values in (a-c) indicate the locations and stream functions of the vortex centers, respectively. 
Fig. 7 Velocity profile at the cross-section $X-X^{\prime}$ of the plug for different plug lengths, (a) The comparison with the 1D model and (b) The effect of the dimensionless plug length $\widehat{L}$.

Fig. 8 Plug resistance coefficient in microcapillaries decreases with the increase of the dimensionless plug length $\widehat{L}$. The symbols are experimental results for air/liquid plug systems by Kreutzer et al. (2005c). The solid curves are the analytical result from the proposed analytical model. The black solid curve is the plug resistance coefficient only considering the contribution of the shear stress. The red, blue, and orange solid curves are the analytical results with fitted constant coefficient $\mathrm{C}$ as shown in Eq. (41) with $\mathrm{C}_{\text {water }}=135.0, \mathrm{C}_{\text {decane }}=83.8$, and $\mathrm{C}_{\text {tetradecane }}=23.8$ respectively. The horizontal dashed line with $\mathrm{C}_{\mathrm{f}}=16$ is plotted to show that the plug resistance coefficient approaches 16 as the dimensionless plug length $\widehat{L}$ approaches infinity.

Fig. 9 Flow patterns in liquid plugs with different contact angles. The interfaces at the advancing and receding ends are assumed to be shapes of spherical caps. Rotational symmetric simulation was carried out via Fluent 6.3.26. Grid independence was tested by the repeating refinement of grid until grid independent solutions are obtained. The control volumes for each plug are around $10^{4}$. 


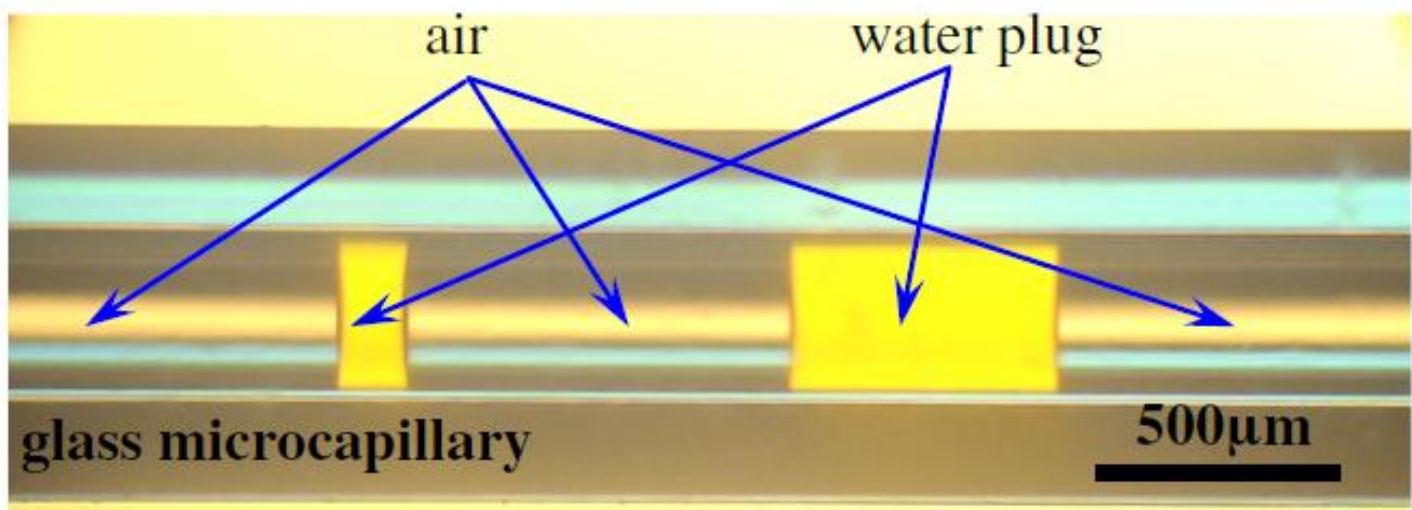

Fig. 1 


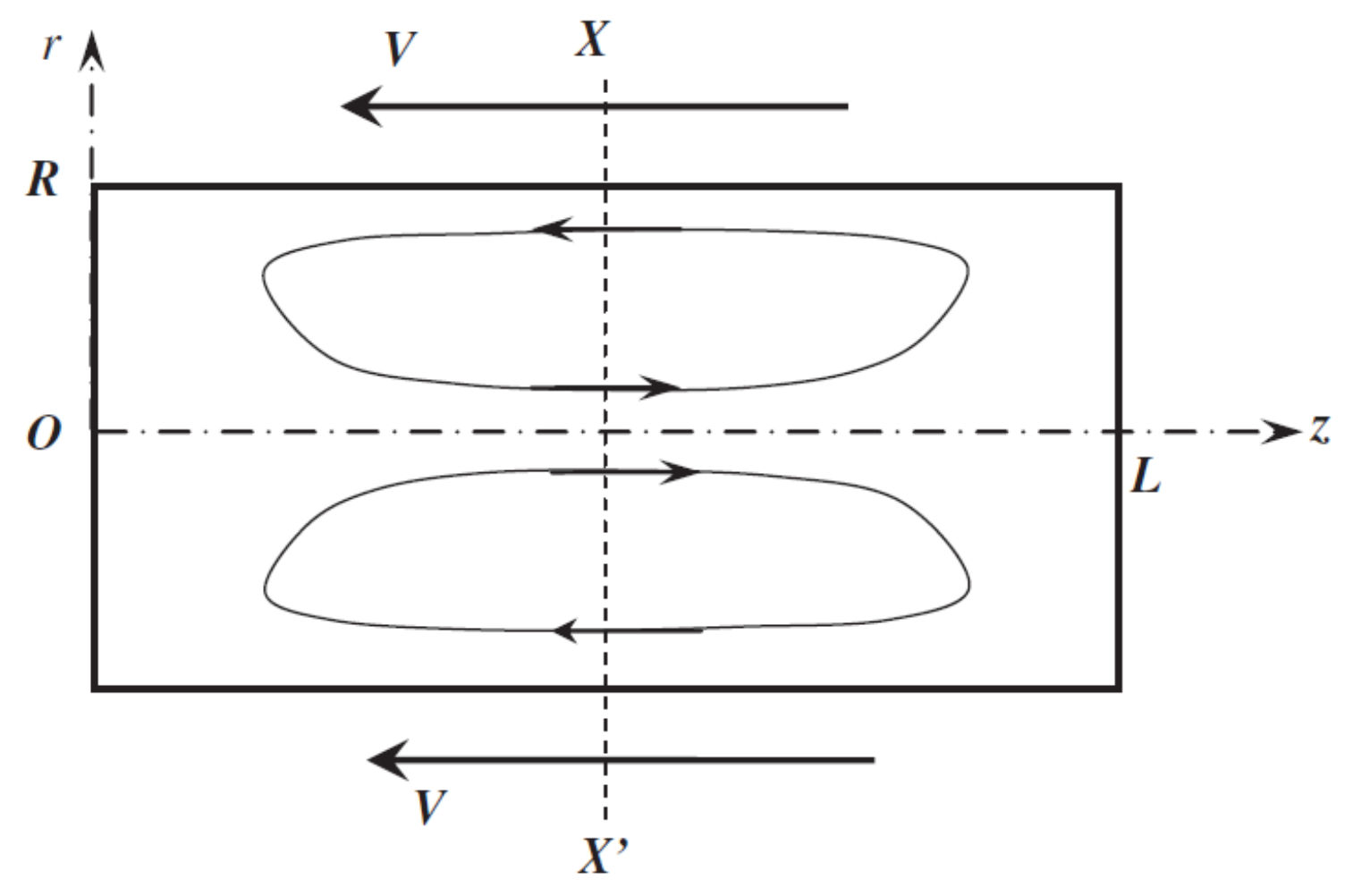

Fig. 2 


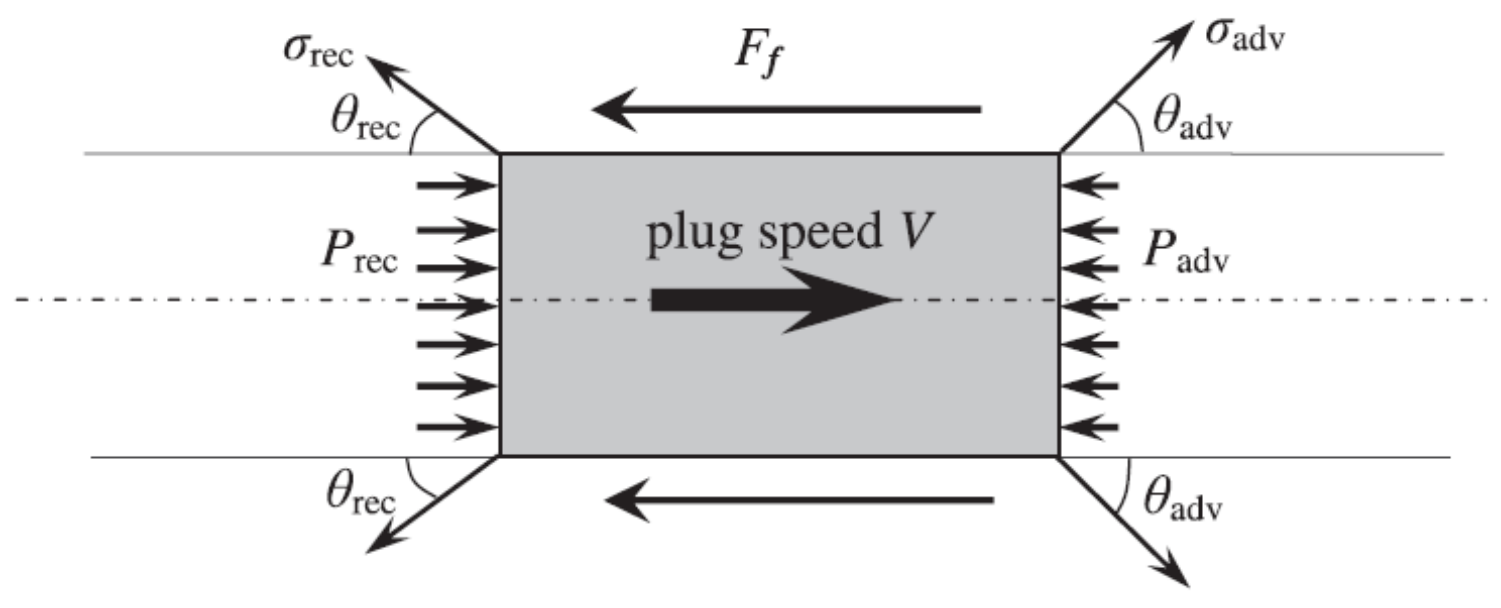

Fig. 3 


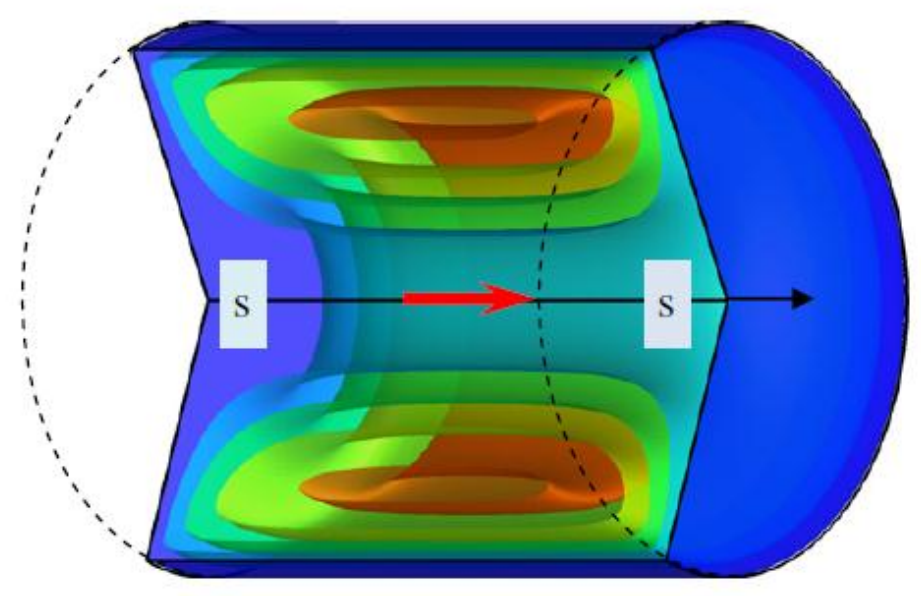

(a)

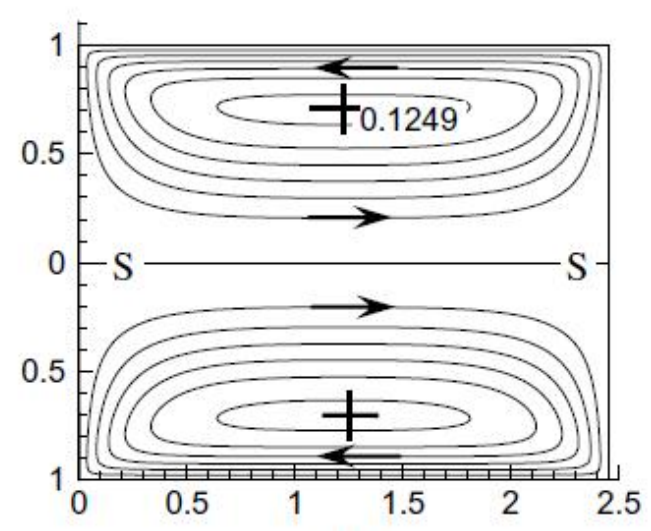

(b)
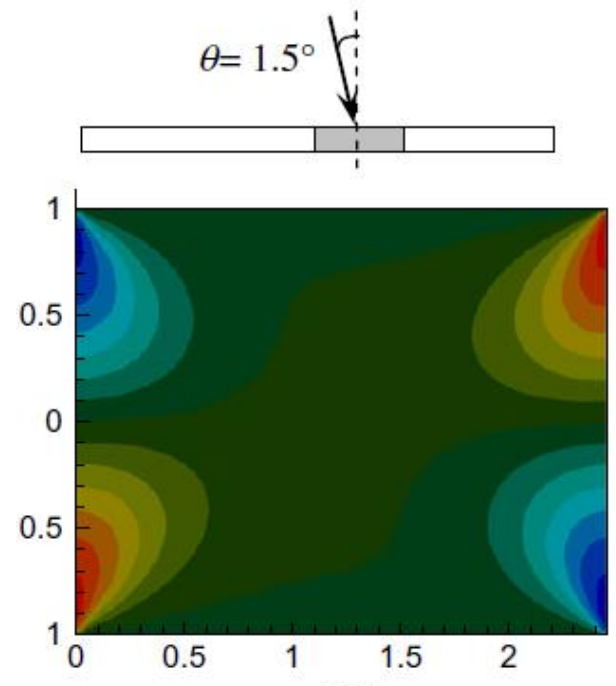

(d)

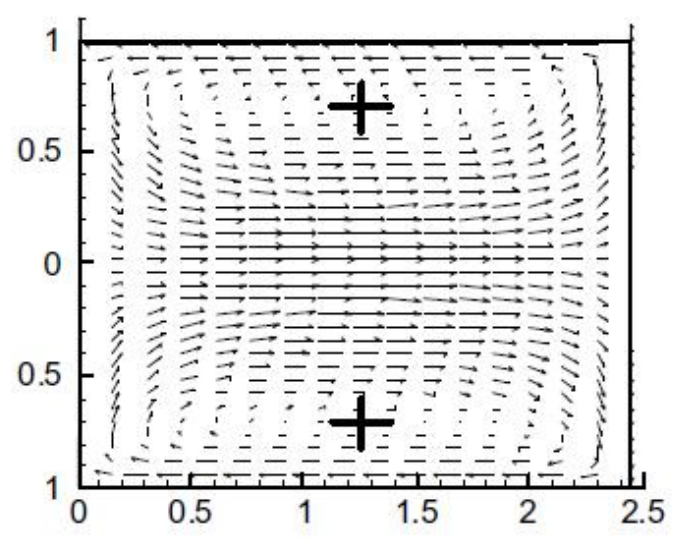

(c)

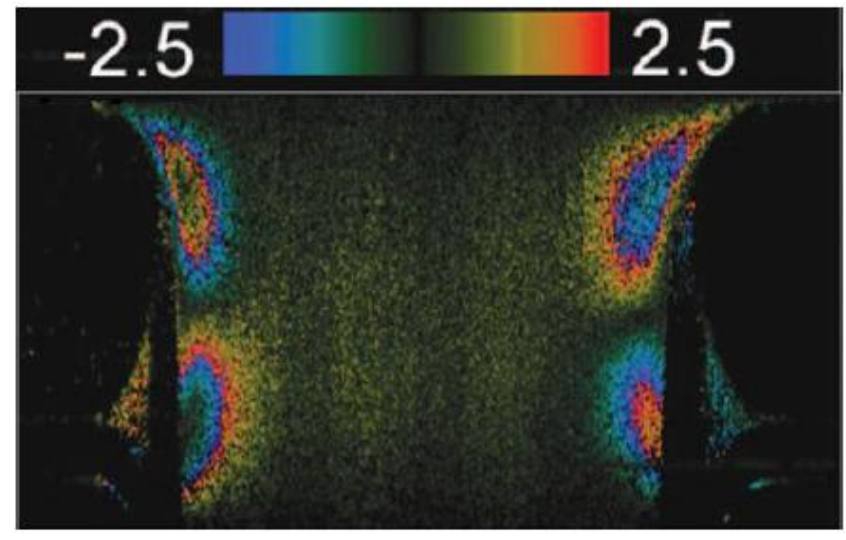

(e)

Fig. 4 


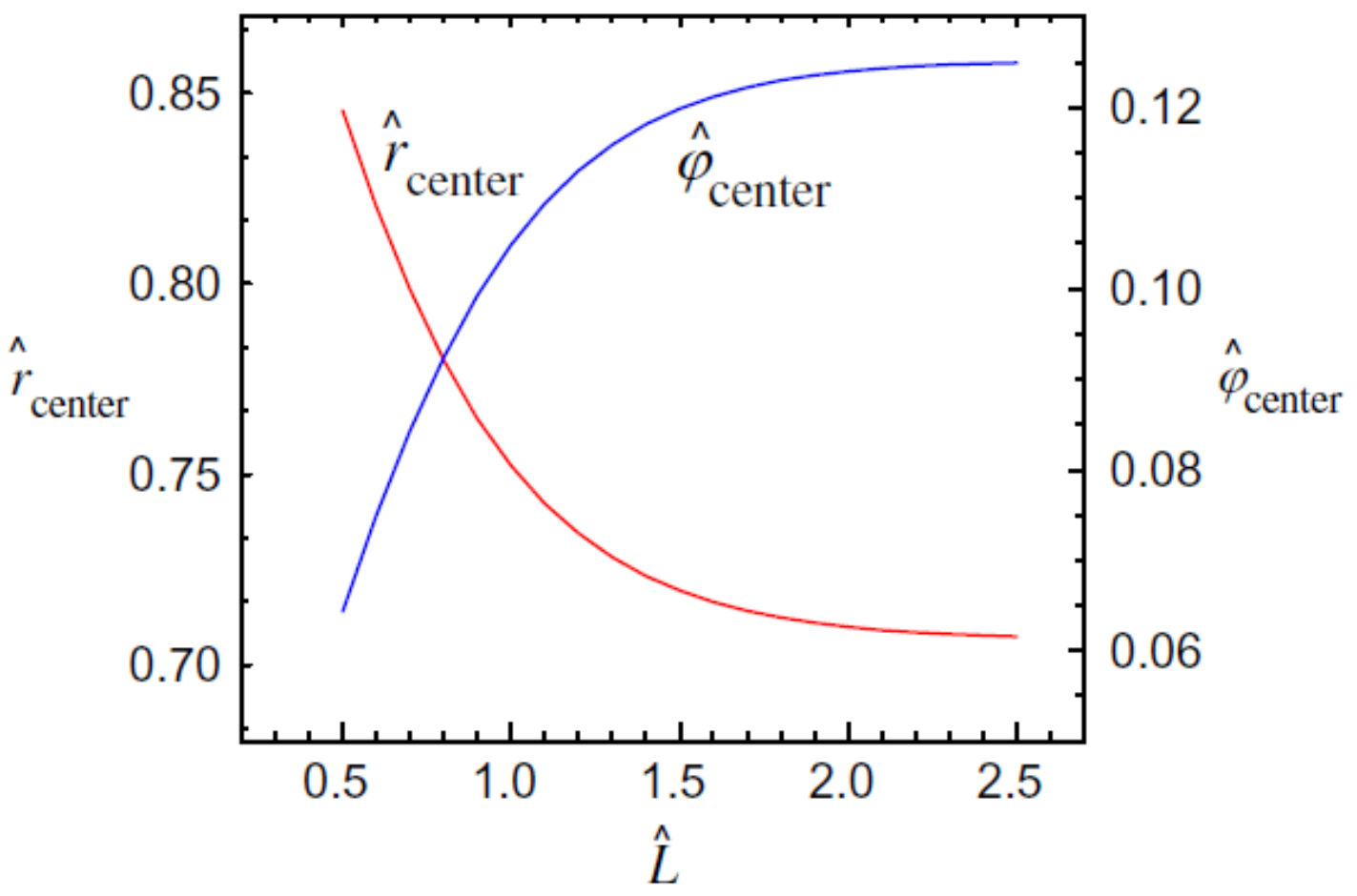

Fig. 5 


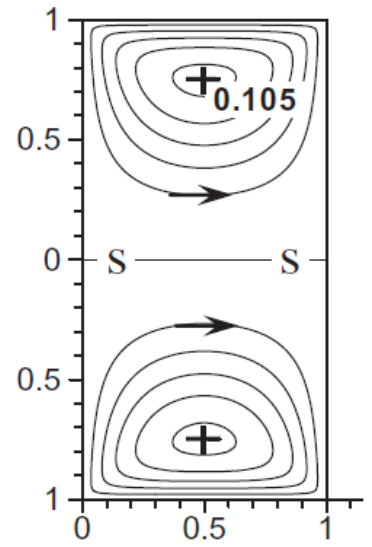

(a)

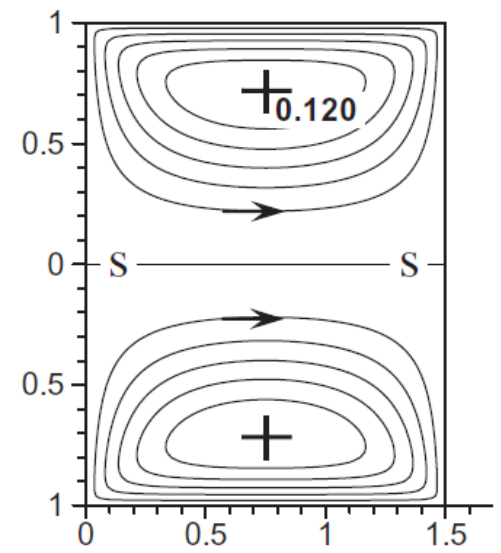

(b)

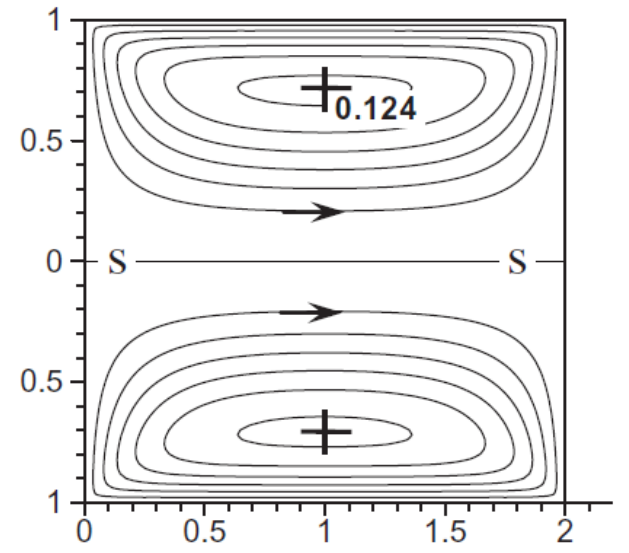

(c)

Fig. 6 

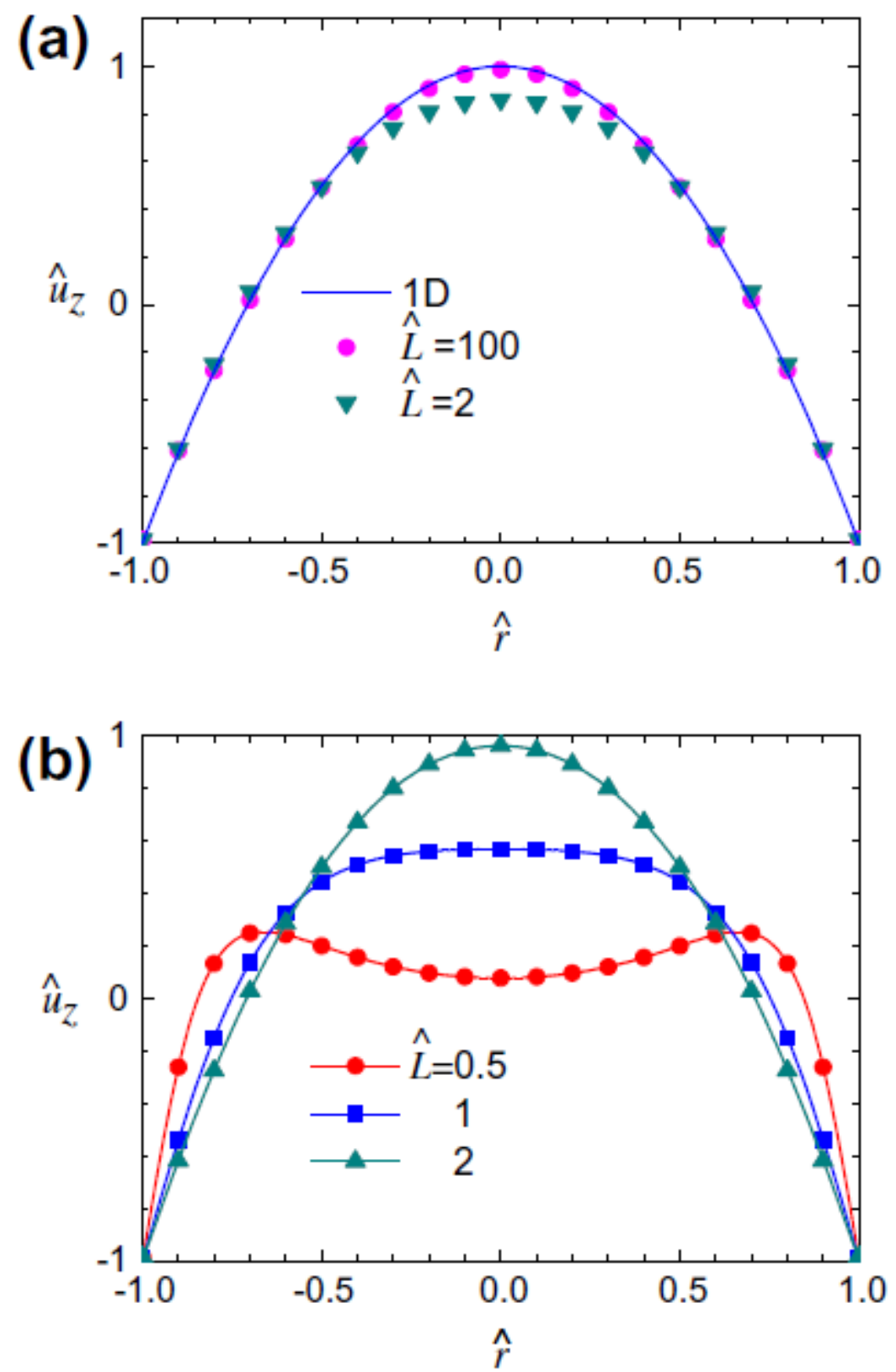

Fig. 7 


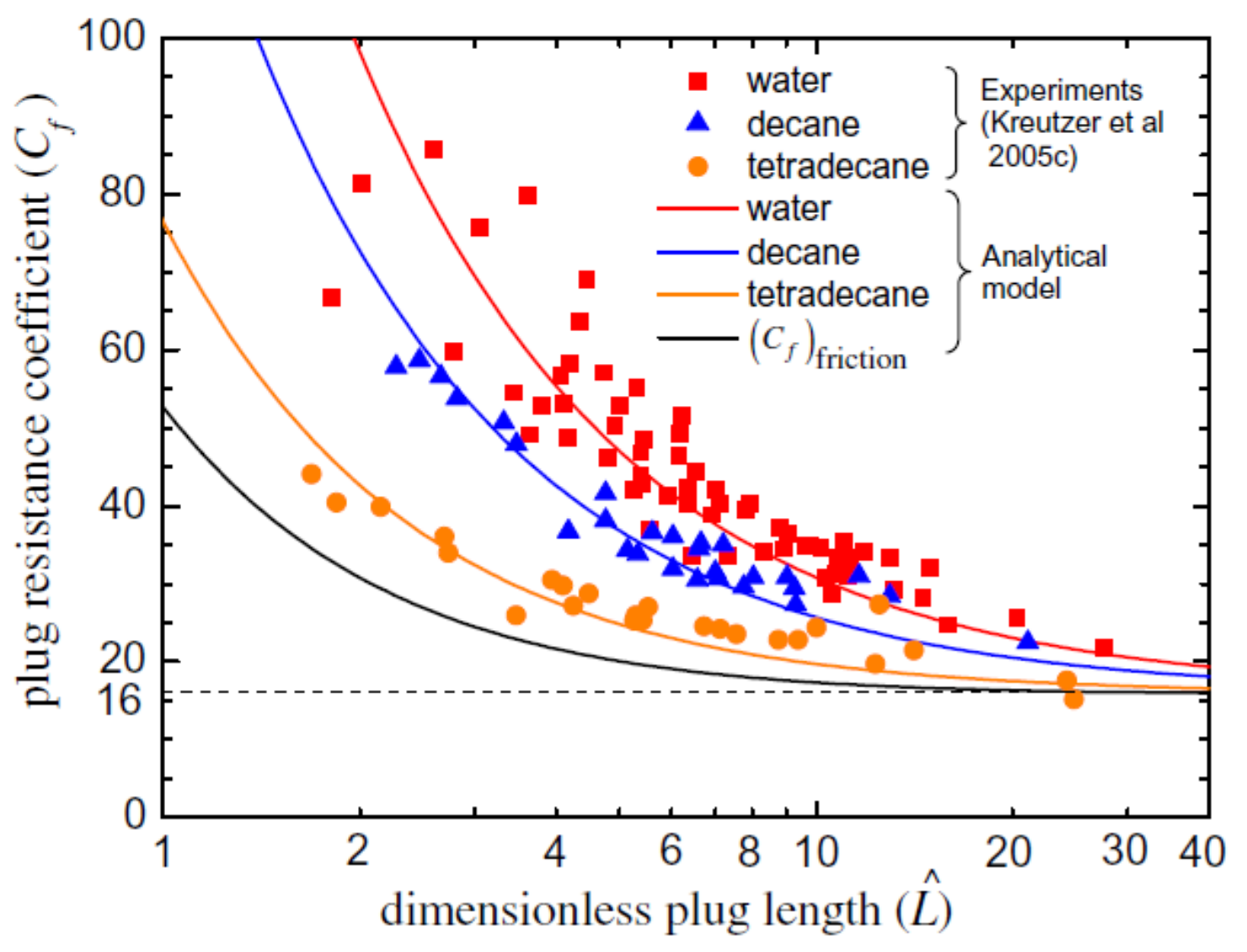

Fig. 8 


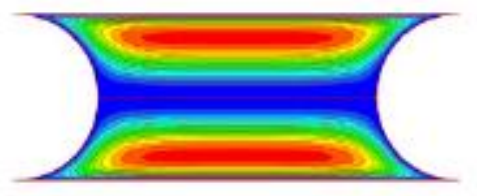

(a) $\theta=0^{\circ}$

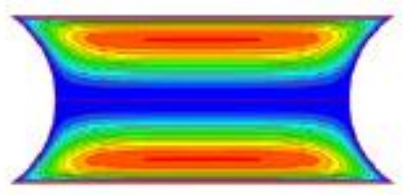

(d) $\theta=33.75^{\circ}$

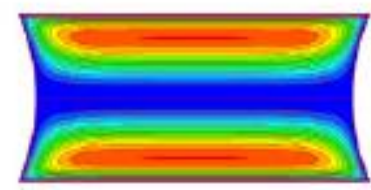

(g) $\theta=67.5^{\circ}$

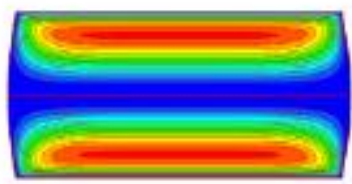

(j) $\theta=101.25^{\circ}$

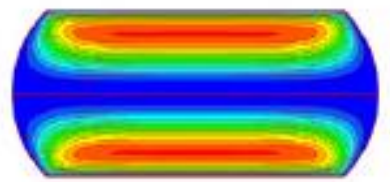

(m) $\theta=135^{\circ}$

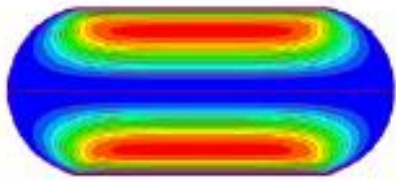

(p) $\theta=168.75^{\circ}$

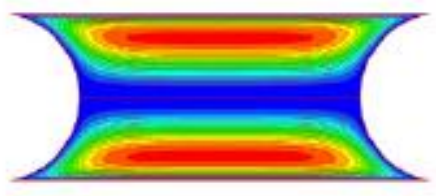

(b) $\theta=11.25^{\circ}$

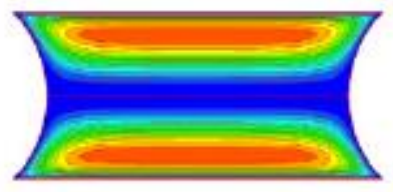

(e) $\theta=45^{\circ}$

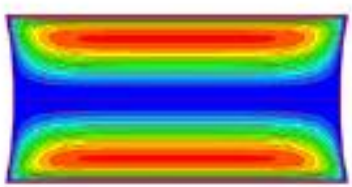

(h) $\theta=78.75^{\circ}$

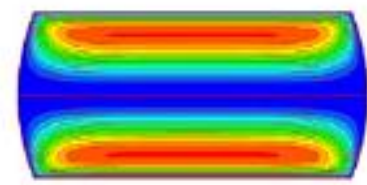

(k) $\theta=112.5^{\circ}$

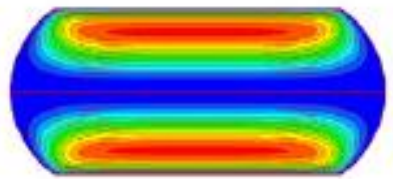

(n) $\theta=146.25^{\circ}$

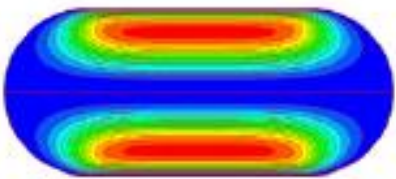

(q) $\theta=180^{\circ}$

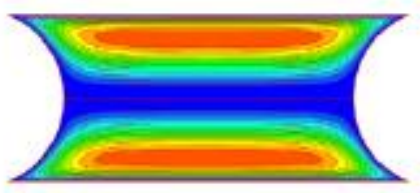

(c) $\theta=22.5^{\circ}$

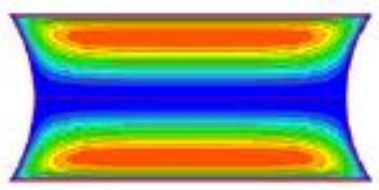

(f) $\theta=56.25^{\circ}$

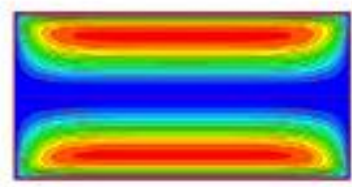

(i) $\theta=90^{\circ}$

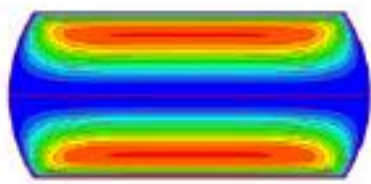

(1) $\theta=123.75^{\circ}$

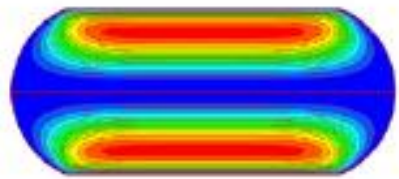

(o) $\theta=157.5^{\circ}$

Fig. 9 